\title{
A MATHEMATICAL MODEL FOR THE DETERMINATION OF VISCOELASTIC BEHAVIOR OF BRAIN IN VIVO-II RELAXATION RESPONSE $\dagger$
}

\author{
HAN CHOU WANG and ALAN S. WINEMAN \\ Highway Safety Research Institute. The University of Michigan. Ann Arbor, Michigan 48104. \\ U.S.A.
}

\begin{abstract}
In a recent experiment for determining the mechanical response of brain in cito a probe, inserted through scaip, skull and dura, is placed in contact with and normal to the brain. given a prescribed motion, and the time variation of corresponding force is measured. In the corresponding continuum mechanical model, brain is idealized as a linear isotropic viscoelastic solid constrained by a rigid skull. At the mating surface. the shear stress and normal displacement vanish everywhere except under the probe which exerts a local radial displacement. This model introduces effective viscoelastic moduli in shear, which is unknown, and in dilatation. which is considered known from other sources. Part II of this study is concerned with stress relaxation induced by a small step displacement of the probe. From the solution of the corresponding quasi-static boundary value problem, a nonlinear Volterra integral equation is established from which the shear stress relaxation function can be solved in terms of measured probe displacement and force. A numerical method of solution is developed.
\end{abstract}

\section{INTRODUCTION}

RECENTLY, Fallenstein et al. (1969) developed an experiment to determine the mechanical response of brain in vivo. A small hole is cut through the scalp, skull and dura of a monkey so that a probe can be put into contact with its brain. After a small preset displacement, the probe is oscillated normal to the brain at a fixed frequency and amplitude. The probe force and displacement are recorded so that their amplitude rate and phase lag can be determined.

The present work is concerned with correlating data from such an experiment with specific material parameters. In order to define the material parameters, a continuum mechanical model must be assumed for the experimental conditions and brain material. In Part I. it was assumed that the skull could be modeled as a rigid spherical container completely filled with a brain modeled as a linear isotropic viscoelastic solid. The probe was modeled as a local normal harmonic displace- ment. By assuming that brain material exhibits linear, isotropic viscoelastic response, two independent material parameters were introduced. One was a frequency dependent shear modulus and the other was a frequency dependent bulk modulus. The bulk modulus is known from other sources (Goldmith, 1966). but the shear modulus is unknown. With the experiment now modeled as a boundary value problem in dynamic linear viscoelasticity. an equation was developed which relates the shear modulus to experimental data. A method of solution was developed and the shear modulus evaluated.

It is the purpose of Part II of this work to study how Fallenstein's probe test can also be used to measure the relaxation response of brain. The relaxation experiment is identical to the oscillatory experiment in all details except that now the probe is subjected to an instantaneously applied displacement which is held constant while time decay of the force required to maintain the probe is measured.

\footnotetext{
$\div$ Received 24 February 1972.

$\div$ Associate professor. Department of Engineering Mechanics.
} 
The relaxation test can be combined with the above mentioned oscillatory test. Some time after the probe has been displaced in the relaxation test the applied force has relaxed to its long term value. The system can now be regarded as having the initial preset required for the oscillatory test.

There are several reasons for doing a relaxation test. First, it is of interest to estimate the order of magnitude of the relaxation modulus and the time for significant amount of stress relaxation. The test is useful for determining the range of linearity of response, since if the probe displacement is altered by a factor of $\alpha$, so should the measured stress. For a linear viscoelastic material, there exists a relation between the shear relaxation modulus and the shear frequency dependent moduius. By determining both moduli experimentally and comparing them against this relation, one can evaluate the assumption of linear viscoelasticity for the mechanical response of brain.

Because the relaxation test is so similar to the oscillatory test. the brain and skull can be modeled as before. The only difference is in the time dependent displacement boundary condition representing the probe. Two independent moduli are again associated with the assumption of linear isotropic viscoelasticity, a time dependent shear relaxation modulus and a time dependent bulk relaxation modulus, the latter as previously mentioned being known. With the relaxation experiment now modeled as a boundary value problem, an equation is developed which relates the shear relaxation function to experimental data. The equation takes the form of a nonlinear Volterra integral equation of the second kind for which a method of solution is developed.

The discussion of the idealization of the experiment into a continuum mechanics model is given in Part I and will only be summarized here for convenience. The model itself and the associated equations are reviewed in Section 2. A general solution to these equations is developed in Section 3. A numerical method for solving the governing integral equations for the shear modulus is discussed in Section 4.

\section{IDEALIZATION OF EXPERIMENT, GOVERNING EQUATIONS}

The general three dimensional form of the constitutive equation for isotropic linear viscoelastic materials depends on two independent material parameters, $\mu(\tau)$ and $\kappa(\tau)$, where $\tau$ denotes time. These are both zero for $\tau<0$ and may have jump discontinuities at $\tau=0 . \mu(\tau)$ represents the relaxation of shear stress due to a shear strain applied at time $\tau=0$ and then maintained constant. $\kappa(\tau)$ represents the relaxation of hydrostatic stress induced by a volumetric strain applied at time $\tau=0$ and then held fixed. Because of this interpretation, $\mu(\tau)$ is called the shear stress relaxation function and $\kappa(\tau)$ is called the bulk relaxation function. According to Goldsmith (1966), the bulk modulus of brain is similar to that of water, a constant whose value is $3 \times 10^{5} \mathrm{psi}$. For use in equation (1), this can be expressed as $k(t)=$ $G_{2}(t) / 3=3 \times 10^{5} 1(t)$, where $1(t)$ is the unit step function, that is, $1(t)=1, t \geqslant 0$ and $1(t)=0, t<0$. For later use, we let $G_{2}(t)=$ $G_{2} 1(t)$, where $G_{2}=9 \times 10^{5}$ psi.

The tensorial stress history $\sigma_{i j}(t)$ corresponding to an arbitrary time varying tensorial strain history $\epsilon_{i j}(t)$ is then given by the constitutive equation

$$
\begin{aligned}
\sigma_{i j}(t)= & G_{1}\left(0^{+}\right) \epsilon_{i j}(t)+\int_{0^{+}}^{t} \epsilon_{i j}(t-\tau) \frac{\partial G_{1}(\tau)}{\partial \tau} \mathrm{d} \tau \\
& +\frac{1}{3} \delta_{i j}\left\{\epsilon_{k k}(t)\left[G_{2}\left(0^{+}\right)-G_{1}\left(0^{+}\right)\right]\right. \\
& \left.+\int_{0^{+}}^{t} \epsilon_{k k}(t-\tau) \frac{\partial}{\partial \tau}\left[G_{2}(\tau)-G_{1}(\tau)\right] \mathrm{d} \tau\right\}
\end{aligned}
$$

where for notational convenience, $G_{1}=2 \mu$, $G_{2}=3 \kappa$. Equation (1) can be written in the following form so as to include the discontinuities of $G_{1}$ and $G_{2}$ at $t=0$ in the integral 


$$
\begin{aligned}
\sigma_{i j}(t)= & \int_{0)^{-}}^{t} \epsilon_{i j}(t-\tau) \mathrm{d} G_{1}(\tau) \\
& +\frac{1}{3} \delta_{i j} \int_{0^{-}}^{t} \epsilon_{k k}(t-\tau) \mathrm{d}\left[G_{2}(\tau)-G_{1}(\tau)\right] .
\end{aligned}
$$

Since its mathematical structure involves both convolution and Stieltjes integration, equation (2) is often abbreviated as (Gurtin and Sternberg, 1962)

$$
\sigma_{i j}=\epsilon_{i j} * \mathrm{~d} G_{1}+\frac{1}{3} \delta_{i j} \epsilon_{k \cdot k} * \mathrm{~d}\left[G_{\underline{2}}-G_{1}\right]
$$

The special notation introduced in equation (3) allows the use of a very convenient algebra for performing operations involving the Stieltjes convolution integral in equation (2). This operator algebra, derived by Gurtin and Sternberg (1962), corresponds only to operations on the time variable and may be interchanged with operations involving spatial variables. These operations will be introduced as needed, but will not be verified.

In constructing a continuum mechanics model of Fallenstein's probe test, the following assumptions are made:

1. Brain tissue will be assumed to be a linear. homogeneous, isotropic viscoelastic material.

2. The brain entirely fills the skull which is assumed to be a spherical case.

3. Since the skull is much harder than the brain tissue, the skull is assumed to be rigid.

4. The excitation load is axisymmetric, which implies that response will also be axisymmetric about an axis through the line of force of the excitation load. Also, displacements occur only in planes through the line of force.

5. Body force is neglected.

6. The connection between the brain and the skull is complex. Generally, there is a thin layer of cerebrospinal fluid between the skull and brain. In addition. there are nerve bundles and blood vessels at the lower part of the brain. Since the probe is placed in a section of the skull far from the restraint of nerve cords, the influence of this is neglected. Furthermore, since the layer of cerebrospinal fluid is thin and its viscosity is small (similar to the water), the additional assumption is made that the shear stress at the interface between brain and skull can be neglected.

Based on the above assumptions, a theoretical model is pictured as shown in Fig. 1 . We introduce spherical coordinates $(R, \theta, \phi)$ with origin at the center of the sphere and with the center line of the probe lying on $\theta=0$.

Assumption 6 means that there is no shear stress at the interface of viscoelastic material and rigid container. Hence the boundary condition on stress takes the form

$$
\sigma_{r \theta}(a, \theta, t)=\sigma_{r \phi}(a, \theta, t)=0
$$

Assumption 4. of axisymmetry, implies $\sigma_{r \phi}=0$, so that the stress boundary condition

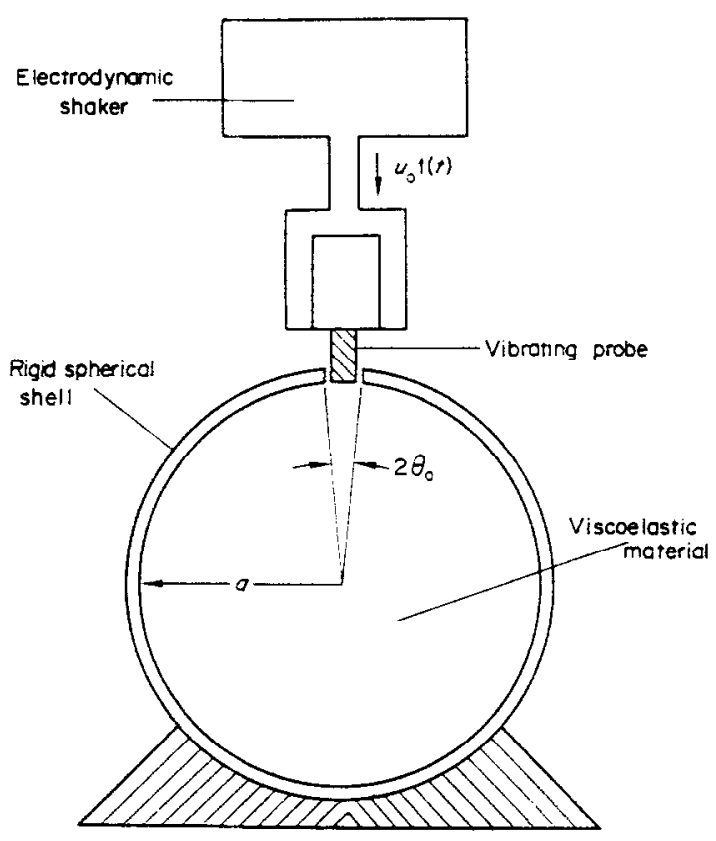

Fig. 1. Model for theoretical analysis. 
reduces to

$$
\sigma_{r \theta}(a, \theta, t)=0 .
$$

Since the skull is assumed rigid, the normal displacement must vanish every where except at the opening provided by the probe. Under the probe the displacement is prescribed. The boundary condition on normal displacement $u_{r}(a, \theta, t)$ then becomes

$$
u_{r}(a, \theta, t)=\left\{\begin{array}{cl}
u(\theta, t) & 0 \leqslant \theta \leqslant \theta_{0} \\
0 & \theta_{0} \leqslant \theta \leqslant \pi .
\end{array}\right.
$$

The normal displacement $u(\theta, t)$ will have its maximum value under the probe, at $\theta=0$, and then decrease gradually as $\theta$ increases until it reaches zero at $\theta=\theta_{0}$. In the relaxation test, the probe is given a radial displacement which varies in time like a step function, i.e.

$$
u(\theta, t)=u(\theta) 1(t)
$$

where $\mathrm{l}(t)=0, t<0$ and $\mathrm{l}(t)=1, t \geqslant 0$.

For notational convenience, the general field equations are stated with respect to a Cartesian coordinate system. It can be assumed that waves generated by this step loading will damp out in a very short period of time, so that the motion may be regarded as quasistatic. Neglecting inertia, the stresses satisfy

$$
\frac{\partial \sigma_{i j}}{\partial x_{j}}=0
$$

For small strains, the strain-displacement equation is

$$
\epsilon_{i j}(\mathbf{X}, t)=\frac{1}{2}\left[\frac{\partial u_{i}(\mathbf{X}, t)}{\partial x_{j}}+\frac{\partial u_{j}(\mathbf{X}, t)}{\partial x_{i}}\right] .
$$

The strain-displacement relations on spherical coordinates are given in Part I and will not be repeated here. For a linear, homogeneous, isotropic viscoelastic material, the equation for the displacement is found from equations (3). (8) and (9) to be

$$
\begin{aligned}
\Gamma^{2} u_{i}(\mathbf{X}, t) \approx \mathrm{d} G_{1}(t)+\frac{1}{3} & \frac{\partial}{\partial x_{i}}\left[\frac{\partial u_{k}}{\partial x_{k}}(\mathbf{X}, t)\right] \approx \mathrm{d}\left[G_{1}(t)\right. \\
\left.+2 G_{1}(t)\right] & =0 \quad(10)
\end{aligned}
$$

or in vector form

$$
\nabla^{3} \mathbf{u} * \mathrm{~d} G_{1}+\frac{1}{3} \nabla(\nabla . \mathbf{u}) \div \mathrm{d}\left(G_{1}+2 G_{2}\right)=0 .
$$

where $G_{1}(t)=2 \mu(t), G_{2}(t)=3 \kappa(t)$.

\section{SOLUTION}

It has been shown by Gurtin and Sternberg (1962) that the general solution of elastostatics in terms of the Papkovich-Neuber displacement potentials can be extended to quasistatic viscoelasticity. That is

$$
\begin{aligned}
\mathbf{u}=\nabla(\Phi+\mathbf{X} \cdot \boldsymbol{\Psi}) & \approx \mathrm{d}\left(G_{1}+2 G_{2}\right) \\
& -4 \Psi \div \mathrm{d}\left(2 G_{1}+G_{2}\right)
\end{aligned}
$$

is the general solution of equation (10). where $\Phi$ and $\boldsymbol{\Psi}$ are, respectively, a scalar valued and a vector valued function of $\mathbf{X}$ and $t$, i.e. $\Phi=$ $\Phi(\mathbf{X}, t), \Psi=\Psi(\mathbf{X}, t)$, which satisfy

$$
\nabla^{*} \Phi=0, \quad \nabla^{2} \Psi=0
$$

Reírring equations (12) and (13) to spherical coordinates, the assumption of axisymmetry implies that $\Phi$ and $\Psi$ depend only on $R, \theta$ and $t$. The additional condition that $u_{\phi}=0$ implies that $\Psi_{\diamond}=0$. If a separation of variables representation for $\Phi$ and $\Psi$ is then assumed, that is, $\Phi$ and $\Psi$ can be expressed in the form $F(R) H(\theta) T(t)$, then the general solution of equation (13) is given by Luré (1964), as

$$
\begin{aligned}
\Phi= & \sum_{n=0}^{\infty}-B_{n} R^{n} P_{n}(\cos \theta) \\
\Psi= & \sum_{n=0}^{\infty}-A_{n}(n+1) R^{n+1} P_{n}(\cos \theta) \mathbf{e}_{r} \\
& +\sum_{n=1}^{\infty} A_{n} R^{n+1} P_{n}^{\prime}(\cos \theta) \mathbf{e}_{\theta}
\end{aligned}
$$

where $A_{n}$ and $B_{n}$ are unknown functions of time. $P_{n}(\cos \theta)$ are Legendre functions and 
$P_{n}^{\prime}(\cos \theta)=\mathrm{d} P_{n} / \mathrm{d} \theta=-P_{n}^{(1)}$, where $P_{n}^{(1)}$ are the associated Legendre functions of order 1.

Substituting the above solutions into equations (12) gives

$$
\begin{gathered}
u_{r}=\sum_{n=0}^{\infty}\left\{-n R^{n-1} B_{n} * \mathrm{~d}\left(G_{1}+2 G_{2}\right)\right. \\
\left.-(n+1) R^{n+1} A_{n} * \mathrm{~d}\left[(n-6) G_{1}+2 n G_{.2}\right]\right\} \\
P_{n}(\cos \theta) \\
u_{\theta}=\sum_{n=1}^{\infty}\left\{-R^{n-1} B_{n} * \mathrm{~d}\left(G_{1}+2 G_{2}\right)\right. \\
\left.-R^{n+1} A_{n} * \mathrm{~d}\left[(n+9)+2(n+3) G_{2}\right]\right\} . \\
P_{n}^{\prime}(\cos \theta) .
\end{gathered}
$$

$A_{n}(t)$ and $B_{n}(t)$ can be determined from boundary conditions of equations $(5-7)$. In order to apply them. note the following;

(i) the radial displacement on the surface in equation (6) can be expressed in Legendre polynomials, i.e.

$u_{r}(a, \theta . t)=u(\theta) 1(t)=\sum_{n=0}^{\infty} c_{n} P_{n}(\cos \theta) 1(t)$

(ii) Equations (1) and (5) imply that $\epsilon_{r \theta}(a$, $\theta . t)$ satisfies

$$
\epsilon_{r \theta}(t) G_{1}(0)+\int_{0}^{\ell} \epsilon_{r \theta}(\tau) \dot{G}_{1}(t-\tau) \mathrm{d} \tau=0 .
$$

where $\dot{G}_{1}$ denotes the derivative of $G_{1}$ with respect to its argument. This is a homogeneous, linear Volterra integral equation of the second kind. If $G_{1}(0) \neq 0$, it has a unique solution (See Smithies, 1958),

$$
\epsilon_{r \theta}(a, \theta, t)=0, t \geqslant 0 .
$$

Using equation (17), a relation between $A_{n}(t)$ and $B_{n}(t)$ is established

$$
\epsilon_{r \theta}(a, \theta)=\left.\left(\frac{1}{r} \frac{\partial u_{r}}{\partial \theta}+\frac{\partial u_{\theta}}{\partial r}-\frac{u_{\theta}}{r}\right)\right|_{r=a}
$$

$$
\begin{aligned}
= & \sum_{n=1}^{x}\left\{-2 a^{n} A_{n} * \mathrm{~d}\left[\left(n^{2}+2 n-3\right) G_{1}\right.\right. \\
& \left.+2 n(n+2) G_{2}\right]-2(n-1) a^{n-2} \\
& \left.\times B_{n} \div \mathrm{d}\left(G_{1}+2 G_{2}\right)\right\} P_{n}^{\prime}(\cos \theta)=0 .
\end{aligned}
$$

Since the associated Legendre functions are linearly independent, for $n=1$,

$$
12 a A_{1} * \mathrm{~d} G_{2}=0
$$

which in integral form is (See equation 11)

$$
12 a\left[G_{2}(0) A_{1}(t)+\int_{0}^{t} A_{1}(\tau) \dot{G}_{2}(t-\tau) \mathrm{d} \tau\right]=0 .
$$

Since $G_{2}(0) \neq 0$, equation $(20)$ is a homogeneous linear Volterra integral equation of the second kind for $A_{1}(t)$. As mentioned above, this equation has the unique solution

$$
A_{1}(t) \equiv 0
$$

$B_{1}(t)$ will be determined later.

For $n \geqslant 2$. the relation between $A_{n}$ and $B_{n}$ is

$$
\begin{aligned}
B_{n} * \mathrm{~d}\left(G_{1}+2 G_{2}\right)= & -a^{2} A_{n} * \mathrm{~d}\left[(n+3) G_{1}\right. \\
& \left.+\frac{2 n(n+2)}{n-1} G_{2}\right]
\end{aligned}
$$

This represents an integral equation for $B_{n}(t)$ in terms of $A_{n}(t)$. Substituting equations (21) and (22) into (15) gives

$$
\begin{aligned}
u_{r}= & 6 R A_{0} \div \mathrm{d} G_{1}-B_{1} * \mathrm{~d}\left(G_{1}+2 G_{2}\right) P_{1}(\cos \theta) \\
& +\sum_{n=2}^{\infty}\left\{a^{2} n R^{n-1} A_{n} \div \mathrm{d}\left[(n+3) G_{1}\right.\right. \\
& \left.+\frac{2 n(n+2)}{n-1} G_{2}\right]-(n+1) R^{n-1} A_{n} \cdots \\
& \left.\times \mathrm{d}\left[(n-6) G_{1}+2 n G_{2}\right]\right\} P_{n}(\cos \theta)
\end{aligned}
$$




$$
\begin{aligned}
u_{\theta}= & -B_{1} * \mathrm{~d}\left(G_{1}+2 G_{2}\right) P_{1}^{\prime}(\cos \theta) \\
& +\sum_{n=2}^{\infty}\left\{a ^ { 2 } R ^ { n - 1 } A _ { n } * \mathrm { d } \left[(n+3) G_{1}\right.\right. \\
& \left.+\frac{2 n(n+2)}{n-1} G_{2}\right]-R^{n+1} A_{n} * \mathrm{~d}\left[(n+9) G_{1}\right. \\
& \left.\left.+2(n+3) G_{2}\right]\right\} P_{n}^{\prime}(\cos \theta) .
\end{aligned}
$$

Evaluating the radial displacement $u_{r}$ on the surface and equating to equation (16) gives

$$
\begin{aligned}
\left.u_{r}\right|_{r=a}= & 6 a A_{0} * \mathrm{~d} G_{1}-B_{1} * \mathrm{~d}\left(G_{1}+2 G_{2}\right) \\
& \times P_{1}(\cos \theta)+\sum_{n=2}^{\infty} a^{n+1} A_{n} * \mathrm{~d}\left[(8 n+6) G_{1}\right. \\
& \left.+2 n\left(\frac{2 n+1}{n-1}\right) G_{2}\right] P_{n}(\cos \theta) \\
= & \sum_{n=0}^{\infty} c_{n} P_{n}(\cos \theta) 1(t) .
\end{aligned}
$$

Due to the linear independence of Legendre functions, $A_{0}$ can be solved from equation (24) as

$$
A_{0} * \mathrm{~d} G_{1}=\frac{c_{0}}{6 a} 1(x)
$$

In integral form, equation (25) becomes

$$
G_{1}(0) A_{0}(t)+\int_{0}^{t} A_{0}(\tau) \dot{G}_{1}(t-\tau) \mathrm{d} \tau=\frac{c_{0}}{6 a} 1(t) .
$$

The integral equation for $B_{1}(t)$ is found from equation (24) to be

$$
-B_{1} \approx \mathrm{d}\left(G_{1}+2 G_{2}\right)=c_{1} 1(t)
$$

or

$$
\begin{aligned}
& {\left[G_{1}(0)+2 G_{2}(0)\right] B_{1}(t)} \\
& +\int_{0}^{t} B_{1}(t-\tau)\left[\dot{G}_{1}(\tau)+2 \dot{G}_{2}(\tau)\right] \mathrm{d} \tau=-c_{1} 1(t) .
\end{aligned}
$$

Equation (24) also gives integral equations for $A_{n}$ for $n \geqslant 2$,

$$
\begin{aligned}
A_{n}(t) * \mathrm{~d}\left[(8 n+6) G_{1}\right. & \left.+2 n\left(\frac{2 n+1}{n-1}\right) G_{2}\right] \\
& =c_{n} 1(t) / a^{n+1} .
\end{aligned}
$$

It was mentioned in Section 2 that $G_{2}(t)=$ $G_{2} 1(t)$, where $G_{2}$ is a known constant. Equation (29) then has the integral form

$$
\begin{gathered}
A_{n}(t)\left[G_{1}(0)+\frac{n}{4 n+3}\left(\frac{2 n+1}{n-1}\right) G_{2}\right] \\
+\int_{0}^{t} A_{n}(t-\tau) \dot{G}_{1}(\tau) \mathrm{d} \tau \\
\quad=c_{n} 1(t) /\left[(8 n+6) \cdot a^{n+1}\right] .
\end{gathered}
$$

If the material parameters $G_{1}, G_{2}$ and the boundary condition $u(\theta)$ are given, one can determine $A_{n}(t)$ from equations (25) and (29), which are all Volterra integral equations of the second kind. Then $B_{n}(t)$ are found by using equations (22) and (28).

After having determined these functions, the stress components will be easily obtained from the stress-strain relations equation (3). These are found to be

$$
\sigma_{r r}=6 A_{0} * \mathrm{~d} G_{1} * \mathrm{~d} G_{2}+\sum_{n=2}^{\infty}\left\{a^{2} n(n-1) R^{n-2}\right.
$$

$$
\begin{aligned}
& \times A_{n} * \mathrm{~d}\left[(n+3) G_{1}+\frac{2 n(n+2)}{n-1} G_{2}\right] * \mathrm{~d} G_{1} \\
& +R^{n} A_{n} * \mathrm{~d} G_{1} * \mathrm{~d}\left[\left(-n^{3}+n\right) G_{1}\right. \\
& \left.\left.-\left(2 n^{3}-8 n-6\right) G_{2}\right]\right\} P_{n}(\cos \theta)
\end{aligned}
$$$$
\sigma_{\theta \theta}=6 A_{0} * \mathrm{~d} G_{1} * \mathrm{~d} G_{2}-\frac{B_{1} * \mathrm{~d} G_{1} * \mathrm{~d}\left(G_{1}+2 G_{2}\right)}{R}
$$$$
\times\left[P_{1}(\cos \theta)+P_{1}^{\prime \prime}(\cos \theta)\right]
$$$$
+\sum_{n=2}^{\infty} R^{n} A_{n} * \mathrm{~d} G_{1} * \mathrm{~d}\left[\left(6 n^{2}+12 n+6\right) G_{2}\right.
$$$$
\left.-\left(3 n^{2}+15 n+12\right) G_{1}\right] P_{n}(\cos \theta)
$$ 


$$
\begin{aligned}
& +\sum_{n=2}^{\infty} a^{2} R^{n-2} A_{n} * \mathrm{~d} G_{1} * \mathrm{~d}\left[\left(n^{2}+3 n\right) G_{1}\right. \\
& \left.+\frac{2 n^{2}(n+2)}{n-1} G_{2}\right] P_{n}(\cos \theta) \\
& +\sum_{n=2}^{\infty}\left\{a ^ { 2 } R ^ { n - 2 } A _ { n } * \mathrm { d } G _ { 1 } * \mathrm { d } \left[(n+3) G_{1}\right.\right. \\
& \left.+\frac{2 n^{2}(n+2)}{n-1} G_{2}\right]-R^{n} A_{n} * \mathrm{~d} G_{1} * \mathrm{~d} \\
& \left.\times\left[(n+9) G_{1}+2(n+3) G_{2}\right]\right\} P_{n}^{\prime \prime}(\cos \theta) \\
& \sigma_{\infty \Phi}=6 A_{9} * \mathrm{~d} G_{1} * \mathrm{~d} G_{2}-\frac{B_{1} * \mathrm{~d} G_{1} * \mathrm{~d}\left(G_{1}+2 G_{2}\right)}{R} \\
& \times\left[P_{1}(\cos \theta)+\cot \theta P_{1}^{\prime}(\cos \theta)\right] \\
& +\sum_{n=2}^{\infty} R^{n} A_{n} * \mathrm{~d} G_{1} * \mathrm{~d}\left[\left(6 n^{2}+12 n+6\right) G_{2}\right. \\
& \left.-\left(3 n^{2}+15 n+12\right) G_{1}\right] P_{n}(\cos \theta) \\
& +\sum_{n=2}^{\infty} a^{2} R^{n-2} A_{n} * \mathrm{~d} G_{1} * \mathrm{~d}\left[\left(n^{2}+3 n\right) G_{1}\right. \\
& \left.+\frac{2 n^{2}(n+2)}{n-1} G_{2}\right] P_{n}(\cos \theta) \\
& +\sum_{n=2}^{\infty}\left\{a ^ { 2 } R ^ { n - 2 } A _ { n } * \mathrm { d } G _ { 1 } * \mathrm { d } \left[(n+3) G_{1}\right.\right. \\
& \left.+\frac{2 n(n+2)}{n-1} G_{:}\right]-R^{n} A_{n} * \mathrm{~d} G_{1} * \mathrm{~d}\left[(n+9) G_{1}\right. \\
& \left.\left.+2(n+3) G_{2}\right]\right\} \cot \theta P_{n}^{\prime}(\cos \theta) \\
& \sigma_{r \theta}=\sum_{n=1}^{\infty}\left\{-2 R^{n} A_{n} * \mathrm{~d}\left[\left(n^{2}+2 n-3\right) G_{1}\right.\right. \\
& \left.+2 n(n+2) G_{2}\right]-2(n-1) R^{n-2} \\
& \left.\times B_{n} * \mathrm{~d}\left[G_{1}+2 G_{2}\right]\right\} \approx \mathrm{d} G_{1}, P_{n}^{\prime}(\cos \theta) .
\end{aligned}
$$

be computed as either $\left(A_{0} * \mathrm{~d} G_{\mathrm{i}}\right) * \mathrm{~d} G_{2}$ or $A_{0} *\left(\mathrm{~d} G_{1} * \mathrm{~d} G_{2}\right)$.

\section{NLMERICAL APPROACH}

If the force or stress under the probe are known from measurements in this relaxation test, the relaxation function $G_{1}(t)$ can be obtained by a numerical method. The stress under the probe will be (from equation 31 )

$$
\begin{aligned}
\sigma_{r r}(a, 0, t)= & 6 A_{0} * \mathrm{~d} G_{1} * \mathrm{~d} G_{2} \\
& +\sum_{n=2}^{\infty} a^{n} A_{n} * \mathrm{~d} G_{1} * \mathrm{~d}\left[\left(2 n^{2}-2 n\right) G_{1}\right. \\
& \left.+\left(4 n^{2}+8 n+6\right) G_{2}\right] .
\end{aligned}
$$

Using equations (26) and (30), equation (32) becomes

$$
\begin{aligned}
\sigma_{r r}(a, 0, t)= & \frac{c_{0} G_{2} 1(t)}{a}+\sum_{n=2}^{\infty} a^{n}\left[\frac{c_{n} 1(t)}{(8 n+6) a^{n+1}}\right. \\
& \left.-\frac{n}{4 n+3}\left(\frac{2 n+1}{n-1}\right) G_{2} A_{n}(t)\right] \\
& \times * \mathrm{~d}\left[\left(2 n^{2}-2 n\right) G_{1}\right. \\
& \left.+\left(4 n^{2}+8 n+6\right) G_{2}\right] .
\end{aligned}
$$

Applying equation (30) again. the above equation reduces to

$$
\begin{aligned}
\sigma_{r r}(a, 0, t)= & \frac{c_{0} G_{2} l(t)}{a}+\sum_{n=2}^{\infty}\left[F_{1}(n) A_{n}(t) G_{2}^{2}\right. \\
& \left.+F_{2}(n) G_{2} 1(t)+F_{3}(n) G_{1}(t)\right]
\end{aligned}
$$

where

$$
\begin{aligned}
F_{1}(n)= & {\left[\frac{4 n^{2}(2 n+1)}{8 n+6}-\left(4 n^{2}+8 n+6\right)\right] } \\
& \times \frac{2 n(2 n+1) a^{n}}{(8 n+6)(n-1)} \\
F_{2}(n)= & {\left[4 n^{2}+8 n+6-\frac{4 n^{2}(2 n+1)}{8 n+6}\right] } \\
& \times \frac{c_{n}}{(8 n+6) a}
\end{aligned}
$$




$$
F_{3}(n)=\frac{2 n(n-1)}{(8 n+6) a} c_{n} .
$$

In deriving these results use was made of the representation $G_{2}(t)=G_{2} 1(t)$ and the operation $A_{n}(t) * \mathrm{~d} 1(t)=A_{n}(t)$. Equation (33) relates the measured stress $\sigma_{r r}(a, 0, t)$ to $G_{1}(t)$. which appears explicitly and also implicitly in the unknown functions $A_{n}(t)$. In order to find $G_{1}(t)$, equation (33) will have to be solved simultaneously with the integral equations (30) for $A_{n}(t)$. The procedure for doing this combines a numerical method for solving integral equation of Volterra type (30) with the graphical procedure outlined in Part I.

Equation (30) can be rewritten as

$$
\begin{aligned}
G_{1}(0) A_{n}(t) & +\int_{0}^{t} A_{n}(t-\tau) \dot{G}_{1}(\tau) \mathrm{d} \tau \\
& =g_{1}(n) 1(t)-g_{2}(n) A_{n}(t)
\end{aligned}
$$

where

$$
\begin{aligned}
& g_{1}(n)=\frac{c_{n}}{(8 n+6) a^{n+1}} \\
& g_{2}(n)=\frac{G_{2} n(2 n+1)}{(4 n+3)(n-1)} .
\end{aligned}
$$

If $t=0+$, equation (35) becomes

$$
G_{1}(0) A_{n}(0)=g_{1}(n)-g_{2}(n) A_{n}(0)
$$

or

$$
A_{n}(0)=g_{1}(n) /\left[G_{1}(0)+g_{2}(n)\right]
$$

Substituting equation (36) into (33) evaluated at $t=0+$ yields

$$
\begin{aligned}
\sigma_{r r}\left(a, 0,0_{+}\right)=\frac{c_{0} G_{2}}{a} & +\sum_{n=2}^{\infty}\left[\frac{F_{1}(n) g_{1}(n)}{G_{1}(0)+g_{2}(n)}\right. \\
& \left.+F_{2}(n) G_{2}+F_{3}(n) G_{1}(0)\right] .
\end{aligned}
$$

From the above equation, a curve of $\sigma_{r r}(a, 0,0)$ vs. $G_{1}(0)$ can be obtained as shown on Fig. 2 .
From this curve, one may find the correct value of $G_{1}(0)$ for which $\sigma_{r r}(a, 0,0)$ will match the experimental results.

If we now let $t=t_{1}>0$, equation (35) becomes

$$
\begin{aligned}
G_{1}(0) A_{n}\left(t_{1}\right)+\int_{0}^{t_{1}} A_{n}\left(t_{1}-\tau\right) G_{1}(\tau) \mathrm{d} \tau & \\
& =g_{1}(n)-g_{2}(n) A_{n}\left(t_{1}\right) .
\end{aligned}
$$

For sufficiently small $t_{1}$, the integral can be approximated using the trapezoidal rule and $\dot{G}_{1}(t)$ can be approximated by a simple finite difference scheme so that the integral equation can be approximated as

$$
\begin{aligned}
G_{1}(0) A_{n}\left(t_{1}\right)+\frac{A_{n}\left(t_{1}\right)+A_{n}(0)}{2} & \cdot\left[G_{1}\left(t_{1}\right)-G_{1}(0)\right] \\
= & g_{1}(n)-g_{2}(n) A_{n}\left(t_{1}\right)
\end{aligned}
$$

from which the following expression for $A_{n}\left(t_{1}\right)$ is obtained

$$
A_{n}\left(t_{1}\right)=\frac{g_{1}(n)-\frac{A_{n}(0)}{2}\left[G_{1}\left(t_{1}\right)-G_{1}(0)\right]}{g_{2}(n)+\frac{G_{1}(0)+G_{1}\left(t_{1}\right)}{2}} .
$$

The right side of equation (38) has only one unknown $G_{1}\left(t_{1}\right)$, since $A_{n}(0)$ and $G_{1}(0)$ have already been found from the previous step. Substituting equation (38) into (33), one will

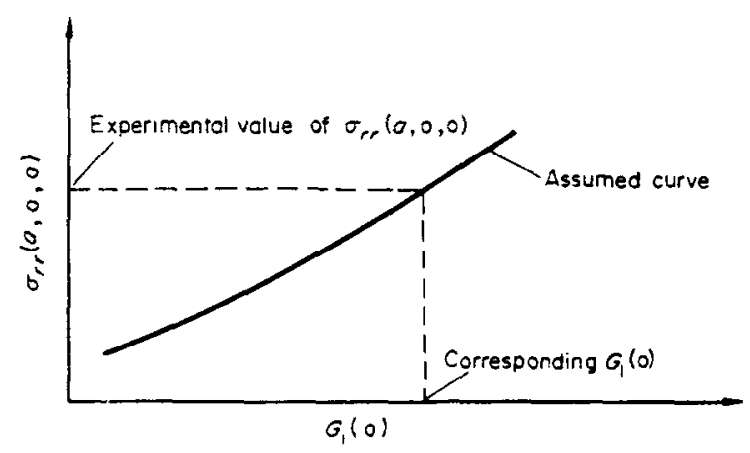

Fig. 2. Possible variation of $\sigma_{r r}(a, 0,0)$ with $G_{1}(0)$. 
get a form similar to equation (37), or

$$
\sigma_{r r}\left(a, 0, t_{1}\right)=\sum_{n=2}^{\infty} f_{n}\left[G_{1}\left(t_{1}\right)\right]+\frac{c_{1} G_{2} 1(t)}{a}
$$

where $f_{n}\left[G_{1}\left(t_{1}\right)\right]$ is a function of $G_{1}\left(t_{1}\right)$ and $n$ for $n=2.3,4$. ... Again a curve of $\sigma_{r r}(a, 0$, $\left.t_{1}\right)$ is. $G_{1}\left(t_{1}\right)$ is obtained. Then for a given $\sigma_{r r}\left(a, 0, t_{1}\right)$, a desired $G_{1}\left(t_{1}\right)$ can be determined from this curve.

Now let $t=t_{2}>t_{1}$, equation (35) can be written as

$$
\begin{aligned}
G_{1}(0) A_{n}\left(t_{2}\right) & +\int_{0}^{t_{1}} A_{n}\left(t_{2}-\tau\right) \dot{G}_{1}(\tau) \mathrm{d} \tau \\
& +\int_{t_{1}}^{t_{2}} A_{n}\left(t_{2}-\tau\right) \dot{G}_{1}(\tau) \mathrm{d} \tau \\
& =g_{1}(n)-g_{2}(n) A_{11}\left(t_{2}\right)
\end{aligned}
$$

If one assumes equal time steps. So that $t_{2}-t_{1}=t_{1}$, equation (40) is approximated by

$$
\begin{gathered}
G_{1}(0) A_{n}\left(t_{2}\right)+\frac{A_{n}\left(t_{2}\right)+A_{n}\left(t_{1}\right)}{2}\left[G_{1}\left(t_{1}\right)-G_{1}(0)\right] \\
+\frac{A_{n}\left(t_{1}\right)+A_{n}(0)}{2}\left[G_{1}\left(t_{2}\right)-G_{1}\left(t_{1}\right)\right] \\
=g_{1}(n)-g_{2}(n) A_{n}\left(t_{2}\right)
\end{gathered}
$$

Continuing as before. this is substituted into equation (33) and the curve of $\sigma_{r r}\left(a, 0, t_{1 .}\right)$ vs $G_{1}\left(t_{2}\right)$ is generated, from which the desired value of $G_{1}\left(t_{2}\right)$ can be found.

In general, let $t=t_{j}>t_{j-1}$. equation (35) will be

$$
\begin{aligned}
& G_{1}(0) A_{n}\left(t_{j}\right)+\int_{0}^{t_{1}} A_{n}\left(t_{j}-\tau\right) \dot{G}_{1}(\tau) \mathrm{d} \tau \\
& +\int_{t_{1}}^{t_{2}} A_{n}\left(t_{j}-\tau\right) \dot{G}_{1}(\tau) \mathrm{d} \tau \\
& +\cdots+\int_{t_{i-1}}^{t_{j}} A_{n}\left(t_{j}-\tau\right) \dot{G}_{1}(\tau) \mathrm{d} \tau \\
& \quad=g_{1}(n)-g_{2}(n) A_{n}\left(t_{j}\right)
\end{aligned}
$$

Again using equal time steps, $t_{j}-t_{j-1}=t_{1}$, equation (43) becomes approximated by

$$
\begin{gathered}
G_{1}(0) A_{n}\left(t_{j}\right)+\frac{A_{n}\left(t_{j}\right)+A_{n}\left(t_{j-1}\right)}{2}\left[G_{1}\left(t_{1}\right)-G_{1}(0)\right] \\
+\frac{A_{n}\left(t_{j-1}\right)+A_{n}\left(t_{j-2}\right)}{2}\left[G_{1}\left(t_{2}\right)-G_{1}\left(t_{1}\right)\right] \\
+\cdots+\frac{A_{n}\left(t_{1}\right)+A_{n}(0)}{2}\left[G_{1}\left(t_{j}\right)-G_{1}\left(t_{j-1}\right)\right] \\
=g_{1}(n)-g_{2}(n) A_{n}\left(t_{j}\right) .
\end{gathered}
$$

From equation (44) one finds $A_{n}\left(t_{j}\right)$ in terms of $G_{1}\left(t_{j}\right)$ and previously determined values of $A_{n}$ and $G_{1}$, i.e.

$$
A_{n}\left(t_{j}\right)=\frac{g_{1}(n)+\frac{A_{n}\left(t_{j-1}\right)}{2}\left[G_{1}(0)-G_{1}\left(t_{2}\right)\right]+\frac{A_{n}\left(t_{j-2}\right)}{2}\left[G_{1}\left(t_{1}\right)-G_{1}\left(t_{3}\right)\right]+\cdots+\frac{A_{n}(0)}{2}\left[G_{1}\left(t_{j-1}\right)-G\left(t_{j}\right)\right]}{G_{1}(0)+g_{2}(n)+\left[G_{1}\left(t_{1}\right)-G_{1}(0)\right] / 2} .
$$

which can be solved for the unknown value of Continuing the process. one obtains $G_{1}\left(t_{j}\right)$, $A_{n}$ at time $t .$. $j=1,2,3, \ldots$, and hence the relaxation func-

$$
A_{n}\left(t_{2}\right)=\frac{g_{1}(n)+\frac{A_{n}\left(t_{1}\right)}{2}\left[G_{1}(0)-G_{1}\left(t_{2}\right)\right]+\frac{A_{n}(0)}{2}\left[G_{1}\left(t_{1}\right)-G_{1}\left(t_{2}\right)\right]}{G_{1}(0)+g_{2}(n)+\left[G_{1}\left(t_{1}\right)-G_{1}(0)\right] / 2} .
$$




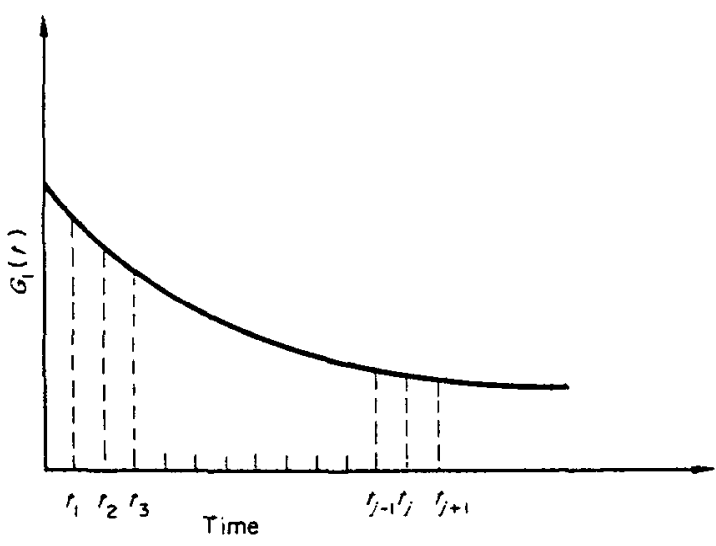

Fig. 3. Construction of the shear stress relaxation function $G_{1}(t)$.

tion in shear $G_{1}(t)=2 \mu(t)$, (See Fig. 3).

It should be emphasized that the method of solution described here is only procedural. In order to obtain better accuracy or to reduce computation, it may be more appropriate to choose logarithmic time steps or a different means of approximating the integrals.

Acknowledgement - This investigation was supported by the Biomechanics Department of the Highway Safety Research Institute of The University of Michigan under contract No. PH-43-67-1136 sponsored by the National Institute of Neurological Diseases and Stroke.

\section{REFERENCES}

Fallenstein. G. T., Hulce. V. D. and Melvin. J. W. (1969) Dynamic mechanical properites of human brain tissue. J. Biomechanics 2, 217-236.
Goldsmith. W. (1966). The physical processes producing head injuries. In Head Injury (Edited by W. F. Caveness and A. E. Walker) p. 283 Lippincott, Philadelphia.

Gurtin. M. E. and Sternberg. E. (1962). On the linear theory of viscoelasticity. Archs ration. Mech. Analysis $11,29 !-356$.

Luré, A. E. (1964) Three-dimensional Problems of the Theory of Elasticity. pp. 325-338. Interscience Publishers, a division of John Wiley and Son, New York.

Smithies. E. (1958) Integral Equations. p. 301, Cambridge University Press, London.

\section{NOMENCLATLRE}

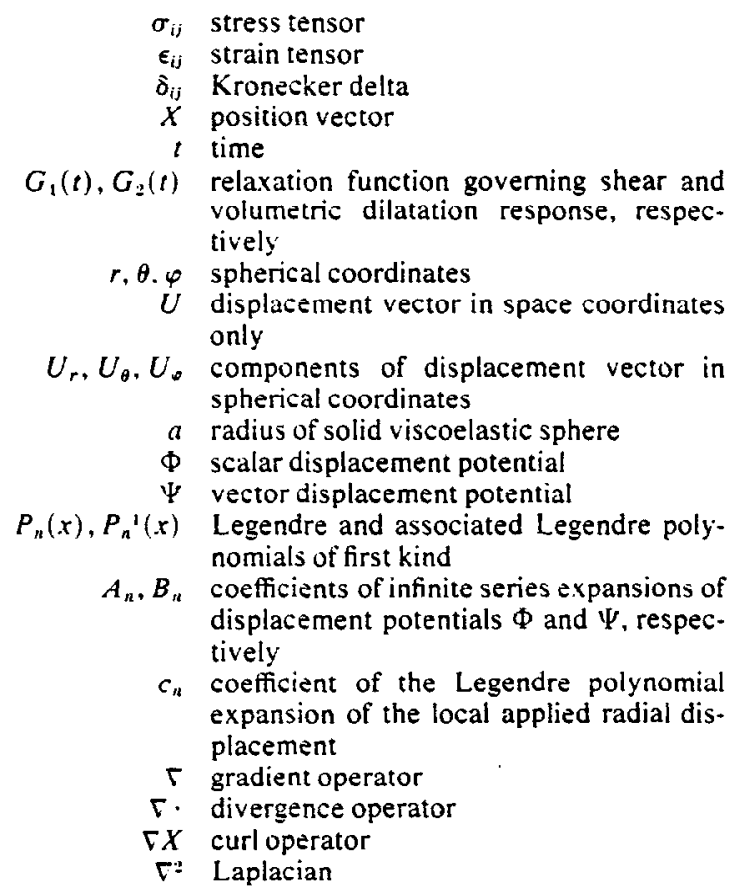

